\title{
Los discursos de lo público y su alcance político
}

\section{The discourses of the public and their political reach}

Leandro Paolicchi*

\begin{abstract}
Resumen: El trabajo se extiende en un breve examen del lugar asignado por algunos autores como Jürgen Habermas, Karl-Otto Apel o Julio De Zan al discurso como instancia de legitimidad y crítica meta institucional de la sociedad. Haciendo un repaso de posiciones contrarias como las de Arnold Gehlen o Niklas Luhmann se procura establecer los alcances y los límites del espacio público - topos natural del discursocomo lugar paradigmático para interrelaciones sociales de fuerte contenido normativo orientadas hacia la validez de las instituciones. Por último, se realizan una serie de precisiones que son necesarias para esa reivindicación del espacio público político.
\end{abstract}

Palabras claves: discurso, sociedad, espacio público, legitimidad, normatividad

\begin{abstract}
This paper extends in a brief examination of the place assigned to discourse by some authors such as Jürgen Habermas, Karl-Otto Apel or Julio De Zan as an instance of legitimacy and meta institutional critic of society. A review of opposing positions such as Arnold Gehlen or Niklas Luhmann seeks to establish the scope and limits of public space - natural place of discourse- as a paradigmatic place for social interrelations of strong normative content oriented towards the validity of institutions. Finally, a series of precisions are made that are necessary for the claim of the importance of the public political space.
\end{abstract}

Key words: discourse, society, public space, legitimacy, normativity

\footnotetext{
* Doctor en Filosofía (UNLa - Argentina). Docente en la Universidad Nacional de Mar del Plata e Investigador Asistente del CONICET. Como becario del DAAD, ha realizado estudios doctorales (2012) y postdoctorales (2014) en la Goethe Universität de Frankfurt am Main bajo la dirección de Axel Honneth. Es autor de los libros "Discurso y Facticidad" (Mar del Plata, Suárez, 2009) y "Praxis, sentido y normatividad. Hacia una reconstrucción pragmática de las acciones humanas" (Buenos Aires, Prometeo, 2014). Dirección electrónica: leandropaolicchi@gmail.com
} 
La Modernidad ha iniciado un proceso que desde un comienzo se ha configurado como aparición y posterior desarrollo de diferentes sistemas dentro de la sociedad. Dentro de este transcurso, hay quienes han señalado, por ejemplo, Hannah Arendt, la "pérdida" o, al menos, "tergiversación" del sentido del espacio público político, tergiversación que se habría iniciado ya con los pensadores de la antigüedad. ${ }^{1}$ La sociedad civil -espacio entre lo privado y el Estado - permaneció siempre asociada a lo político estatal y nunca terminó de instituir al espacio público como instancia de la asociación política y de preocupación por la cosa pública. ${ }^{2}$

Asimismo, junto con ese proceso de progresiva autonomización de los subsistemas sociales se ha ido configurando un mapa en el cual la antigua jerarquía de la praxis de la polis ha sido desbancada a favor de una horizontalización de los subsistemas. El camino que va desde la política aristotélica como culminación de la praxis colectiva en la antigüedad -con un característico sentido normativo ${ }^{3}$ a la Modernidad, tiene como resultado el desmontaje de la vieja pirámide en el cual la política se termina ubicando como uno más de entre todos los subsistemas. Esta horizontalización se ha reforzado por el desarrollo de una dinámica y un lenguaje técnico propio en cada uno de estos subsistemas. Esto puede verse de un modo paradigmático en Niklas Luhmann en donde los sistemas, no solo tienen su propio código, sino que han devenido autopoiéticos.

${ }^{1}$ De Zan, Julio, La vieja y la nueva politica. Libertad, podery discurso. Buenos Aires, UNSAM Edita, 2013, p. 84.

${ }^{2}$ Benhabib, Seyla, "Modelos de Espacio Público. Hannah Arendt, la tradición liberal y Jürgen Habermas", en El Ser y el Otro en la ética contemporánea, Barcelona, Gedisa, 2006, p. 107.

${ }^{3}$ Habermas, Jürgen, Teoría y Praxis, Madrid, Tecnos, 1988, pp. 49-86; Hennis, Wilhem, Politica y Filosofía Práctica, Buenos Aires, SUR, 1973, pp. 20-27 y Böhler, Dietrich, "Instrumentelle und praktische Vernunft - das ethische Dilemma der Neuzeit" en Apel, K.-O., Böhler, D. und Rebel, K., Funkeolleg Praktische Philosophie / Ethik, Studientexte, Wienheim y Basilea, Beltz, t. II, 1984, pp. 396-433. 
En este proceso consagrado por la Modernidad, la pérdida de primacía de la política frente a otros ámbitos como la economía o la ciencia ha sido acompañada por la pérdida del sentido normativo de la teoría y, por lo tanto, de su transición también a la praxis. La política debe renunciar, según las teorías que, como la de Luhmann, socavan todo intento de normativismo, a intervenir en la dinámica propia de los otros subsistemas y marcar un posible rumbo para el conjunto social. La política es ahora uno más en el conjunto de los subsistemas sociales.

Sin embargo, para ciertos autores este proceso de desarrollo progresivo de los subsistemas sociales no es un proceso inocuo ni tampoco irreversible. Es decir, el desarrollo de los subsistemas hace que la convivencia entre ellos no sea estable. El hiperdesarrollo del sistema económico y la hipertrofia del burocrático administrativo terminan imponiéndose sobre los otros subsistemas y subsumiéndolos bajo la lógica del poder y del dinero. Frente a este proceso, ciertos autores que se autocomprenden como continuadores de la tradición moderna, pero que no resignan la orientación normativista propia de la premodernidad, buscaron revertirlo instalando a la teoría ético-política como instancia de alumbramiento de principios normativos, así como una revalorización del ámbito de las interrelaciones cotidianas comprendido bajo el mundo de la vida. Como un núcleo institucionalizado dentro de este terreno, la sociedad civil y el espacio público se constituyen en la columna vertebral de un proyecto político que busca retener algo del contenido normativo de la democracia, tal como es posible observarlo en la obra de Julio de Zan.

\section{El espacio público como topos natural del discurso}

En esta revalorización del mundo de la vida confluyen planteos profundamente críticos de la Modernidad como los provenientes de la postmodernidad, posturas ético-políticas como las de los comunitaristas y planteamientos que, si bien se ubican como críticos de la Modernidad, creen que un núcleo racional de ésta debe ser preservado. La Modernidad habría desarrollado, para estos últimos autores, sólo uno de sus aspectos principales mientras que habría olvidado otro de sus impulsos originales. Este es el caso 
de Karl-Otto Apel y, sobre todo, de Jürgen Habermas para quien la Modernidad es todavía un proyecto incompleto que debe ser todavía llevado a cabo y que algunos de sus logros, como la noción de una moral universalista o un concepto de autonomía individual, deben ser preservados.

Esta revalorización del mundo de la vida frente al avance de la racionalidad instrumental de los subsistemas sociales se ha producido, como se dijo, porque se vio en él el ámbito paradigmático de un tipo de interrelación que podría funcionar a modo de control de la racionalidad desbocada de los subsistemas sociales y del cual se podría extraer un contenido normativo que funcionara a modo de integración de la totalidad de lo social -punto que para el funcionalismo sistémico siempre constituyó un problema- y también un criterio normativo desde el cual criticar la "colonización" del mundo de la vida.

El tipo de interrelación que caracteriza al mundo de la vida aparece vertebrado por la comunicación lingüística, comunicación que se diferencia de los lenguajes altamente técnicos de los subsistemas por su comprensión llana, es decir, lo que comúnmente entenderíamos por lenguaje ordinario. ${ }^{4}$ Estas formas de interrelación que caracterizan al mundo de la vida se adensan en ciertos ámbitos como la familia y sobre todo en el espacio público político. El espacio público es una estructura comunicativamente organizada y enraizada en el mundo de la vida. ${ }^{5}$

El espacio público, tal como Habermas lo describe, no representa un sistema y como tal no puede concebírselo como una institución con diferencias de roles y con grados de competencia. ${ }^{6}$ Aquí el espacio público es una red para la comunicación de opiniones y es en esencia de horizontes difusos, inestables y porosos. En tanto enraizado en el mundo de la vida el espacio público se estructura en base a un tipo de acción orientada al entendimiento, la acción comunicativa en términos habermasianos, y por lo tanto solo requiere el conocimiento del lenguaje ordinario, típico de la práctica comunicativa cotidiana.

\footnotetext{
${ }^{4}$ Habermas, Jürgen, Facticidad y V alider, Madrid, Trotta, 1998, p. 434.

${ }^{5}$ Ibidem, p. 439.

${ }^{6}$ Ibidem, p. 440.
} 
La importancia de la esfera pública dentro de la arquitectónica de Habermas no es menor, más bien recae sobre ella la fuente última de legitimación de la dominación política en su totalidad. El espacio público político es la fuente originaria de lo que Habermas llama, tomando un concepto presente ya en Arendt, poder comunicativo. Es decir, el poder que surge cuando los hombres actúan en común y se ponen de acuerdo públicamente sobre algo. ${ }^{7}$ Es sobre este poder, movilizado en el espacio público, sobre el que se hace depender la producción y legitimación de las leyes y, a través de éstas, el control de la administración estatal ordenada en torno al código poder. De este modo, la burocracia administrativa queda ligada a través del derecho a esa fuente última de legitimación que es el poder comunicativo asentado en el espacio público político. Cito a Habermas: "A la base del poder de la administración estatal, constituida en términos jurídicos, ha de haber un poder comunicativo productor de derecho, a fin de que no se seque la fuente de justicia, de la que se legitima el derecho mismo." "Por último, la idea de estado de derecho aparece sin más en Habermas como "la exigencia de ligar el poder administrativo, regido por el código poder, al poder comunicativo creador de derecho, y mantenerlo libre de las interferencias del poder social, es decir, de la fáctica capacidad de imponerse que tienen los intereses privilegiados".

Donde también puede percibirse el rol imprescindible que desempaña el espacio público es en la obra de Karl-Otto Apel. Apel ha encaminado todo su pensamiento al bosquejo de una ética universal que ponga en condición a los hombres de asumir la responsabilidad por las consecuencias de sus acciones que, en el estado actual del desarrollo científico tecnológico, no pueden tener sino dimensión planetaria. Esto puede verse muy fácilmente en las discusiones en torno a los desarrollos nucleares de Corea del Norte o Irán o en los protocolos de protección del medio ambiente.

Dicho bosquejo ha pasado en Apel por la explicitación de un principio moral, llamado principio del discurso, que exige la argumentación como principal salida a los conflictos de intereses. Dicho principio, cuya fundamentación consiste en reconstruirlo desde los presupuestos de toda argumentación con

\footnotetext{
${ }^{7}$ Ibidem, p. 215.

${ }^{8}$ Ibidem, p. 214.

${ }^{9}$ Ibidem, p. 218.
} 
sentido, se encuentra para Apel por encima del nivel de las instituciones o el de los subsistemas funcionales de la sociedad. ${ }^{10}$ De hecho, al principio del discurso le corresponde una función meta-institucional en relación con todas las instituciones, es decir, aquí también las aplicación de un principio discursivo se ejerce contra la racionalidad de las instituciones, es decir, se ve a las instituciones como restricción y amenaza de una racionalidad discursiva que se percibe como resguardo de interacciones libres de dominación y como criterio normativo de justicia.

Apel de todos modos no es un pensador cercano a lo utópico y más bien se acerca a cierto realismo cuando acepta de alguna manera la inevitabilidad de algún grado de coerción funcional por parte de las instituciones junto con el momento de auto alienación que ellas implican. Pero junto con el principio del discurso, y como contrapartida de una responsabilidad individual que todos tenemos dentro del marco de las instituciones, Apel cree que existe una corresponsabilidad primordial de todos los hombres por nuestras instituciones. ${ }^{11}$ Cito a Apel:

Se abre la posibilidad de la ya mencionada acción individualmente responsable por debajo y al nivel de las instituciones, y, por otro lado -sobre las instituciones- la posibilidad de la participación corresponsable en el control público y, por así decirlo, meta institucional - es decir, la potencial legitimación o la crítica y, posiblemente, la reforma de las instituciones. ${ }^{12}$

El ámbito paradigmático para el ejercicio de esta corresponsabilidad y para el desempeño de la función meta-institucional del discurso es lo que Apel denomina espacio público mundial o también publicidad mundial raciocinante. Apel, como se dijo, es realista y es consciente de que a este tipo de ámbito le falta obligatoriedad práctica, pero también cree que entre este espacio y el nivel

\footnotetext{
10 Apel, Karl-Otto, "La ética discursiva y las coerciones funcionales sistémicas de la política, el derecho y la economía de mercado. Reflexión filosófica acerca del proceso de globalización” en Damiani, Alberto y Maliandi, Ricardo, ¿Es peligroso argumentar? Estudios sobre política y argumentación, Mar del Plata, Suárez, 2002, pp. 26.

11 Ibidem, p. 38.

12 Ibidem, p. 39.
} 
de las instituciones se ubica un espacio cuasi institucional al que llama el de los "miles de coloquios y conferencias" y que comprende como potenciales miembros a "delegados de todas las naciones y a representantes de todas las profesiones"13 y en el que se tratan todos los temas relevantes de la humanidad como las cuestiones de seguridad militar, los derechos humanos y la protección ambiental. Entonces, para Apel, "la cuasi institución de los miles de coloquios y conferencias es hoy de hecho el ámbito paradigmático de aplicación de la corresponsabilidad primordial de todo hombre por encima del nivel de las instituciones y sus coerciones funcionales". ${ }^{14}$

\section{La irrelevancia del espacio público. Niklas Luhmann y la sociedad como conjunto de subsistemas autopoiéticos}

Mientras que en los planteos de Apel y Habermas el discurso público funciona a modo de piedra de toque de la legitimidad de las instituciones, existen otros planteos que más bien recalcan la impotencia o llanamente la peligrosidad del ejercicio irrestricto de una crítica discursiva de las instituciones. Este es el caso de Niklas Luhmann. Como se dijo, Luhmann se enfila detrás de una comprensión de la sociedad como un complejo de subsistemas, pero a ello le suma la idea de que los subsistemas sociales autónomos que componen la sociedad se vuelven sobre sí mismos y desarrollan sus propios códigos semánticos y se constituyen en entornos los unos para los otros. Estos códigos que los subsistemas desarrollan son los lenguajes específicos y fundamentales para la operatividad dentro de los sistemas y, entre los propios subsistemas, y no las interacciones de los sujetos implicados dentro de los subsistemas.

La política en el sistema luhmanniano, y en esto Luhmann da cuenta de los desarrollos de la Modernidad, deja de tener la primacía como cúspide de la sociedad con su centro en el Estado y pasa a formar parte como uno más de todos los subsistemas. Con esta última constatación, más la idea de que las interacciones de los individuos no son relevantes para el funcionamiento de los sistemas sino sus propios códigos, Luhmann cercena la conexión que la política tenía asignada en Habermas con las interacciones cotidianas del mundo de la

\footnotetext{
13 Ibidem, p. 43.

${ }^{14}$ Ibidem, p. 44.
} 
vida y, con esta desconexión, el sistema de la administración queda fuera del control de un espacio público político y puede reproducirse a sí mismo sin interferencias normativas.

Los individuos, de todas formas, no quedan fuera totalmente de este proceso, pero intervienen de una forma no organizada y no crítica sino bajo la comprensión de una estructura clientelar. La administración regula la política de proyectos gubernamentales y la política, a través de los partidos, organiza el menú para que el público elija. Este último puede tratar de influir, pero lo hace solo a través de los grupos particulares de interés. ${ }^{15}$ Aquí Luhmann se acerca en la descripción de los procedimientos democráticos a través de la óptica de la teoría de los subsistemas a los planteos clásicos de la teoría elitista de la democracia desde Schumpeter hasta Down. Como dice Habermas: "Desde el punto de vista de la teoría de sistemas, el modo de operar del sistema político se mide por la racionalidad de una regulación o control autorreflexivos, que del contenido de la democracia no deja ya nada más que una distribución alternante del poder entre gobierno y oposición."16

La constatación en Luhmann de que en la Modernidad la política deja de ser el centro o la cúspide jerárquica de lo social se transforma en la advertencia permanente de la frustración a la que conduce el concebir lo político como la solución de todos los problemas de la sociedad. Luhmann menciona una y otra vez como un error clásico de las concepciones modernas y viejo europeas el desbordamiento constante al que someten al sistema político. La política sólo puede proveer soluciones para Luhmann en la medida en que puede traducir las demandas a su propio código binario. Así, es la propia política la que determina la medida de sus posibilidades y no las exigencias de otros subsistemas. Sin embargo, Luhmann recalca una y otra vez que en las sociedades actuales se tiende a sobreexigir a los sistemas políticos.

Esto puede verse con claridad en dos casos paradigmáticos. Uno es el problema de la ecología y otro, al que Luhmann ha dedicado un libro, es el Estado de bienestar. Detrás de la estructuración del Estado de bienestar se cristaliza una idea expansiva del Estado que generalmente ha sido criticada por

${ }^{15}$ Luhmann, Niklas, Sociedad y Sistema: la ambición de la teoría, Barcelona, Paidós, 1997, pp. 61-70.

${ }^{16}$ Habermas, Jürgen, Facticidady Valides, p. 411. 
quienes defienden una idea restrictiva de éste. Para Luhmann la concepción expansiva todavía permanece atrapada en esta vieja idea ya mencionada de la política como centro y culminación de la sociedad. La idea restrictiva le lleva una ventaja porque por lo menos es consciente de las limitaciones del sistema político. En general toda idea de planificación macro desde lo político estatal le parece a Luhmann una idea condenada al fracaso porque no es más que la correlación política de la teoría científica, es decir, es la variante tecnocrática del desbordamiento de lo político ${ }^{17}$.

Entonces, la teoría luhmanniana, a diferencia de lo que planteaba Gehlen de la peligrosidad de liberar a las instituciones a una irrestricta crítica y legitimación por parte de una racionalidad discursiva, no sostiene que no deba buscarse la formación de una voluntad colectiva y democrática en torno a ciertos temas en un espacio público, sino que en un nivel empírico describe la inutilidad o la impotencia de semejante proceso. La teoría de sistemas de Luhmann no propone ninguna teoría normativa de la política o de la democracia, sino simplemente destila un escepticismo al describir empíricamente los procesos administrativos y políticos y cuánta incidencia tienen en ellos la formación e intervención de una posible formación democrática de la voluntad.

No hay en Luhmann un espacio asignado a la elaboración de problemas que se refieren al conjunto de lo social. Son los subsistemas particulares los que elaboran los problemas al asimilarlos y retraducirlos a sus propios lenguajes específicos que descartan la utilización del lenguaje ordinario del mundo de la vida. El espacio público político queda vaciado de sentido y función al no representar ya un aporte significativo al tratamiento de los problemas que afectan a la sociedad en su conjunto.

\section{Los límites de la disolución normativa de la política}

${ }^{17}$ Von Beyme, Klaus, Teoría política del siglo XX. De la modernidad a la posmodernidad, Madrid, Alianza, 1994, p. 236. 
Lo primero que podría decírsele a Luhmann con respecto a la crítica de la idea expansionista del Estado es que detrás de la idea de un Estado de bienestar se encuentran ciertas concepciones y principios de justicia social. Luhmann a esto respondería sin titubear dos cosas. Primero, la introducción de criterios morales de justicia en la política no tiene ningún tipo de éxito en el sentido de un cambio en los patrones de conducta de la humanidad, y segundo, en términos funcionales, lo que haría sería generar más peligros a los peligros reales. La moralización de lo político, en el sentido que podía entenderlo Kant, es para Luhmann la más perjudicial de las recomendaciones.

Sin embargo, a esta posición puede finalmente endilgársele el dejarse llevar por un prejuicio epistemológico, a saber, aquel que cree que los saberes de los especialistas de los diferentes subsistemas pueden aislarse de concepciones morales particulares. Esto es aún más evidente cuando de lo que se trata es de cuestiones relevantes para la sociedad. Los saberes y los lenguajes específicos de los expertos de los diferentes subsistemas están impregnados de cuestiones normativas que quedan inmediatamente a la vista cuando en los programas a implementar estallan los conflictos entre los propios expertos. Es decir, lo moral en lo político está presente aun en las perspectivas tecnocráticas.

Otra observación importante que debe hacérsele a Luhmann es que la autonomización de los subsistemas y su creciente desarrollo no conviven en armonía con el conjunto de los derechos fundamentales de las personas. Este es el caso de un modo evidente del sistema económico. En un sentido discurre el desarrollo exponencial de un sector de la población mientras que en sentido contrario una mayoría se pauperiza y se excluye. Es decir, el estado de derecho entra en tensión con la dinámica de los procesos de los subsistemas. Esto demuestra que la lógica de los sistemas tampoco puede quedar totalmente al margen del control de un espacio público político.

La última afirmación nos lleva a otro punto también importante. Más allá de los procesos de la Modernidad en términos de reacomodamiento de las esferas de saber y de acción, la política no es un subsistema como el resto en el sentido de su desarrollo autopoiético y vuelto hacia sí mismo. ${ }^{18} \mathrm{El}$ sistema político, por un lado, mantiene la estructura del estado de derecho y esto puede

${ }^{18}$ Habermas, Jürgen, Facticidady Valider, p. 432. 
hacerlo porque, por otro lado, permanece abierto e insertado en los canales de la formación democrática de la voluntad. Es decir, el sistema político hunde sus raíces y se alimenta de un espacio público político y, a través de éste, del mundo de la vida y de la red de interacciones lingüísticas que lo estructuran.

Esta forma de centralidad de la sociedad civil y del espacio público político para el funcionamiento del sistema democrático ha sido resaltado por De Zan:

El concepto contemporáneo y posliberal comprende ahora a la sociedad civil, por lo contrario, como el espacio central de lo político, el lugar de la formación de la opinión pública y de la voluntad general en el sentido de Rousseau, que tiende a suprimir la alienación política de la ciudadanía y reasumir la soberanía popular. La idea de contrato social se reinterpreta, entonces, también como un procedimiento y proceso discursivo real y actual de formación de consensos sociales y políticos siempre revisables en el marco del consenso constitucional. ${ }^{19}$

Esta centralidad, de todas maneras, no puede afirmarse sin ningún tipo de reparos. El protagonismo de la esfera pública no puede llevarnos, por ejemplo, a una visión negativa tout court de la representación política y del aparato burocrático del Estado. Tampoco debe llevarnos a sobrecargar de expectativas las posibilidades de acción política de los movimientos que se desenvuelven en ese ámbito.

En parte, algunos de estos reparos ya habían sido formulados por Habermas. En efecto, ya en Facticidady V alidez se advierte que estos espacios no deben considerarse el punto donde "se concentran los rayos de una autorregulación de la sociedad en conjunto" 20 pues su capacidad de acción es limitada en términos de alcance de los movimientos que allí se despliegan.

Esta consideración autolimitante tiene su fundamento en tres puntos. Primero, las sociedades civiles solo pueden surgir en el seno de culturas políticas con cierto ejercicio de libertades civiles. Segundo, a los actores que

${ }^{19}$ De Zan, Julio, La vieja y la nueva política, p. 97.

${ }^{20}$ Habermas, Jürgen, Facticidad y Valider, p. 452. 
despliegan sus acciones en estos ámbitos les está dada la injerencia sobre el decurso de la política, pero no ejercen el poder de manera sustancial y directa. Por último, los movimientos de la sociedad civil deben renunciar a operar desde la política como si este subsistema tuviera las viejas facultades transformadoras del todo de lo social. En parte puede controlar los desarrollos de los subsistemas, pero en parte debe también resignarse a dejar inalterados ciertas adquisiciones técnicas de estos.

Es decir, la centralidad de la sociedad civil y el espacio público para el funcionamiento de la democracia debe defenderse, tal como ocurre en Habermas o en De Zan, con algunas precisiones. Dos precisiones más que considero necesarias son: 1) con respecto al tipo de ciudadanía que es posible construir a partir de ellas, y 2) con respecto al desafío que recibe de la avanzada de un discurso neoliberal que parece solaparse con un proyecto político democrático.

Con respecto al primer punto, como bien se ha señalado, ${ }^{21}$ es posible identificar tres formas de construir ciudadanía dentro de la sociedad civil: una ciudadanía crítica pero antipolítica, una ciudadanía participativa y una radical de ruptura. ${ }^{22} \mathrm{Si}$ bien la primera critica la brecha entre representantes y representados y su centro se dirige a una reforma del sistema político entiende la democracia solamente en términos procedimentales ${ }^{23}$ y sostiene que "el mercado electoral sigue siendo el mejor asignador de liderazgos políticos". ${ }^{24}$

Con respecto a la última, aparece centrada en la posibilidad de una ruptura total con el sistema capitalista y en un rechazo de todo lo referente al Estado y al sistema político ${ }^{25}$. Sólo la opción intermedia parece adecuarse al espíritu de lo que buscan defender tanto Habermas como De Zan. Es decir, una ciudadanía que se construye en el espacio público con una reivindicación

${ }^{21}$ García Delgado, Daniel y Nosetto, Luciano, "La ciudadanía en una etapa de reconstrucción: imaginarios y desafíos" en Erasmus. Revista para el diálogo intercultural, VI, 2, 2004, pp. 171-190.

22 Ibidem, pp. 180 y ss.

${ }^{23}$ Ibidem, p. 181.

${ }^{24}$ Ibidem.

${ }^{25}$ Ibidem, p. 185. 
de la política como tal y que apunta a una reconstrucción del Estado. En palabras de García Delgado y Nosetto:

Esta noción del espacio público está nutrida por una perspectiva de la política no en tanto mera administración de la cosa pública, sino en tanto coexistencia en el espacio público de expresiones sociales con recursos diferenciales en pugna por la defensa de determinados proyectos de sociedad y de definiciones del bien común. Es en este marco que la noción de poder cambia dramáticamente, habilitando el pensamiento del poder en tanto relación social y la posibilidad de constituir a partir de la cooperación y la sociabilidad genérica nuevas fuentes de poder. ${ }^{26}$

Es decir, de los tres modelos de ciudadanía sólo este último parece ser adecuado para un control democrático de los procesos políticos. Sólo un concepto de ciudadanía que no descree de la representación política y la política en general y tampoco procura una ruptura total con el Estado puede constituirse en un centro sobre el cual fortalecer la sociedad civil y el espacio público político dentro de ella.

Por último, cabe mencionar el peligro de un solapamiento con cierto discurso neoliberal que hace necesarias ciertas precisiones. Es decir, como se ha remarcado con certeza ${ }^{27}$ existe una reivindicación de la sociedad civil que lo que busca en realidad es una progresiva reducción de los recursos y responsabilidades del Estado para transferirlas hacia sectores de la sociedad civil. ${ }^{28} \mathrm{El}$ resultado de este movimiento, central a los proyectos neoliberales, es la proliferación de ONGs con altas capacidades técnicas y financiadas por agencias internacionales que sólo responden a sus financiadores o al Estado, pero no al espacio público en el cual actúan.

Para finalizar entonces, los roles centrales de la sociedad civil y el espacio público político, tales como los pensaron Habermas y De Zan para constituirse

\footnotetext{
${ }^{26}$ Ibidem, pp. 183 y 184.

27 Dagnino, Evelina, "Sociedad civil, participación, ciudadanía: ¿de qué estamos hablando?", en Erasmus. Revista para el diálogo intercultural, VI, 2, 2004, pp. 285- 306.

${ }^{28}$ Ibidem, p. 287.
} 
como lugares centrales del funcionamiento democrático, sólo pueden hacerlo si se deja en claro qué tipo de ciudadanía se construye dentro de ellos y se los demarca de un discurso neoliberal que también procura ponerlos en el centro, pero con consecuencias falsamente democráticas.

\section{Algunas conclusiones}

Para finalizar entonces, teorías como la de Gehlen, o especialmente la de Luhmann, pueden servir para resaltar la importancia de las instituciones como estabilizadoras de las conductas y las expectativas recíprocas de los individuos. La idea que defiende Luhmann de que la política no puede ser ya la panacea para todos los conflictos sociales puede servir también para efectivamente no sobrecargar al sistema político con exigencias desmedidas que pueden conducir a crisis de legitimación irreversibles y trágicas para el sistema de gobierno. Pero, aun así, no puede ser soslayada esa corresponsabilidad primordial de los hombres para con sus instituciones, que Apel mencionaba como inscripta en el discurso y ejercida a través de él. Sólo en la medida que se da fundamento a esa corresponsabilidad puede mantenerse una estructura de derechos fundamentales de los hombres en el marco de un estado democrático. Sólo en esta arquitectónica bosquejada anteriormente se pueden salvar las aporías de la teoría funcionalista de sistemas que permanece ciega frente al potencial legitimador de los procesos discursivos que tienen su lugar en el espacio público y que buscan su institucionalización a través del sistema político.

\section{Bibliografía}

- Apel, Karl-Otto, "La ética discursiva y las coerciones funcionales sistémicas de la política, el derecho y la economía de mercado. Reflexión filosófica acerca del proceso de globalización”, en Damiani, Alberto y Maliandi, Ricardo (eds), ¿Es peligroso argumentar? Estudios sobre política y argumentación, Mar del Plata, Suárez, 2002, pp. 23-54. 1985.

- Apel, Karl-Otto, La Transformación de la Filosofía, 2 vol., Madrid, Taurus, 
- Benhabib, Seyla, "Modelos de Espacio Público. Hannah Arendt, la tradición liberal y Jürgen Habermas" en El Ser y el Otro en la ética contemporánea, Barcelona, Gedisa, 2006, pp. 105-138.

- Böhler, Dietrich, "Instrumentelle und praktische Vernunft - das ethische Dilemma der Neuzeit" en Apel, K.-O., Böhler, D. und Rebel, K., Funkkolleg Praktische Philosophie / Ethik, Studientexte, Wienheim y Basilea, Beltz, t. II, 1984.

- Dagnino, Evelina, "Sociedad civil, participación, ciudadanía: ¿de qué estamos hablando?” en Erasmus. Revista para el diálogo intercultural, VI, 2, 2004, pp. 285-306.

- De Zan, Julio, La vieja y la nueva politica. Libertad, poder y discurso. Buenos Aires, UNSAM, 2013.

- García Amado, Juan Antonio, La Filosofía del Derecho de Habermas y Lubmann, Bogotá, Universidad Externado de Colombia, 1997.

- García Delgado, Daniel y Nosetto, Luciano, "La ciudadanía en una etapa de reconstrucción: imaginarios y desafíos" en Erasmus. Revista para el diálogo intercultural, VI, 2, 2004, pp. 171-190.

- Gehlen, Arnold, Moral und Hypermoral, Frankfurt am Main, Athenäum, 1973.

- Habermas, Jürgen, Historia y crítica de la opinión pública. La transformación estructural de la vida pública, Barcelona, G. Gili, 1981.

- Habermas, Jürgen, Teoría y Praxis, Madrid, Tecnos, 1988.

- Habermas, Jürgen, El discurso filosófico de la modernidad, Madrid, Tecnos, 1989.

- Habermas, Jürgen, Facticidady Validez, Madrid, Trotta, 1998.

- Hennis, Wilhem, Política y Filosofía Práctica, Buenos Aires, SUR, 1973.

- Luhmann, Niklas, Fin y Racionalidad en los Sistemas, Madrid, Editora Nacional, 1983.

- Luhmann, Niklas, Sociedad y Sistema: la ambición de la teoría, Barcelona, Paidós, 1997. 
- Luhmann, Niklas, Teoría Politica en el Estado de Bienestar, Madrid, Alianza, 1997b.

- Michelini, Dorando, Globalización, Interculturalidad y Exclusión. Ensayos Ético-Políticos, Río Cuarto, Ediciones del ICALA, 2002.

- Schumpeter, Joseph, Capitalismo, Socialismo y Democracia, Barcelona, Folio, 1996.

- Von Beyme, Klaus, Teoría política del siglo XX. De la modernidad a la posmodernidad, Madrid, Alianza, 1994.

Recibido: 02/2019; aceptado: 05/2019 